\title{
EVALUACIÓN DE LA ENSEÑANZA Y EL APRENDIZAJE
}

\section{ARTÍCULO DE REVISIÓN}

PINTO, Jacyguara Costa ${ }^{1}$

RODRIGUES, Helton Barbosa ${ }^{2}$

SILVA, Vanessa de Souza da ${ }^{3}$

PINTO, Jacyguara Costa. RODRIGUES, Helton Barbosa. SILVA, Vanessa de Souza da. Evaluación de la enseñanza y el aprendizaje. Revista Científica Multidisciplinar Núcleo do Conhecimento. año 04, Ed. 11, Vol. 03, págs. 81-90. Noviembre de 2019. ISSN: 2448-0959, Enlace de acceso: https://www.nucleodoconhecimento.com.br/educacion-es/evaluaciondocente

\section{RESUMEN}

El artículo presenta estudios sobre los nuevos desafíos planteados a la educación, y es esencial pensar en el modelo de evaluación utilizado por los profesores hoy en día. Por lo tanto, buscamos analizar los procesos de planificación de evaluación llevados a cabo en los grados iniciales de la escuela primaria. Los resultados se obtuvieron

${ }^{1}$ Doctor en Ciencias de la Educación, Máster en Ciencias de la Educación, Lato Sensu Especialista en Enseñanza de Educación Superior, Lato Sensu Especialista en Historia Cultural, Literatura Africana, Afrobrasileña, Bachiller y Licenciado en Historia. ${ }^{2}$ Master of Science in Education, Full Degree y Bachelor of Geography, Lato Sensu Specialist in Environmental Management, profesor de la red pública estatal, actualmente ejerce sus funciones como profesor de educación básica en el Sistema Organizacional de enseñanza modular del Estado de Amapá.

${ }^{3}$ Estudiante de maestría en ciencias de la educación, especialista en educación especial, pedagogía completa con licencia, profesor de las escuelas públicas estatales y municipales en el estado de Amapá. 
mediante el uso del método de investigación bibliográfica de carácter descriptivo y enfoque cualitativo, realizando el análisis de contenidos de libros y artículos científicos que permitieron conectar los estudios y perspectivas empíricas del investigador, guiando la construcción de los resultados en tres secciones, que fueron descritas a partir de este momento. En la elaboración del artículo, se analizó la evaluación de la enseñanza y el aprendizaje, y se describió la importancia de la autoevaluación para el educador imparcial; y, fue posible comprender brevemente la adopción de la evaluación participativa por objetivos en la realidad educativa de la escuela primaria. Se llegó a la conclusión de que se respondía al problema del artículo, se alcanzaba el objetivo general y se confirmaba la hipótesis de que cualquier instrumento utilizado por los profesores y la escuela para realizar la evaluación, configuraba una forma de establecer niveles de aprendizaje, reconocer lo mejor y lo peor, y sobre todo, adaptar la enseñanza a los estándares de excelencia instalados por la escuela. Las medidas estandarizadas han desviado el sentido democrático, crítico, constructivo y creativo de la evaluación, impidiendo el camino del aprendizaje efectivo.

Palabras clave: Evaluación, competencias, rendimiento, metas, escuela.

\section{INTRODUCCIÓN}

La educación en nuestro país es bastante compleja, la segregación de la sociedad ha destrozado a grupos de personas, siendo víctimas de disparidades que son los reflejos de la incuria del poder público.

Estas disparidades se reflejan en las relaciones que existen en la escuela. Llevando al individuo a cuestionar sus condiciones, como un ser capaz de construir sus concepciones del ser humano, la sociedad y el mundo, en la búsqueda de satisfacer sus necesidades en diversas dimensiones y al mismo tiempo legitimar su identidad ante sus pares.

Dados los nuevos retos que plantea la educación, es esencial pensar en el modelo de evaluación utilizado por los profesores hoy en día, considerando que el acto de evaluar, sea cual sea el modelo adoptado no es neutral, porque trae a su 
protuberancia una cierta forma de concebir la realidad, el individuo y la sociedad, dirigiendo y condicionando la práctica pedagógica existente en el aula y en la escuela.

Cualquiera que sea el instrumento utilizado por los profesores y la escuela para realizar la evaluación, configura ciertos, establece niveles de aprendizaje, reconoce lo mejor y lo peor, y especialmente adapta la enseñanza a los estándares de excelencia instalados por la escuela. Las medidas estandarizadas han desviado el sentido democrático, crítico, constructivo y creativo de la evaluación, impidiendo el camino del aprendizaje efectivo. Sobre la base de estos supuestos, la investigación comienza con el siguiente problema: ¿Qué relación existe entre las acciones de planificación de la evaluación escolar con la mejora del rendimiento educativo del estudiante?

Así, se justifica reflexionar sobre los procesos que implican la planificación de la evaluación escolar como un momento de aprendizaje, un medio en el que el profesor utiliza para mediar en conocimiento y no un fin, ya que la evaluación no se limita sólo a la determinación de las calificaciones, sino que sigue el camino que el estudiante sigue, descubre sus dificultades y cambia las direcciones si es necesario.

Así, el objetivo del artículo era analizar los procesos de planificación de evaluación llevados a cabo en los grados iniciales de la escuela primaria. Específicamente, se pretende analizar la evaluación de la enseñanza y el aprendizaje; describir la importancia de la autoevaluación para el educador imparcial; y, para entender la adopción de la evaluación participativa por objetivos en la realidad educativa de la escuela primaria.

Los resultados se obtuvieron mediante el uso del método de investigación bibliográfica de carácter descriptivo y enfoque cualitativo, realizando el análisis de contenidos de libros y artículos científicos que permitieron conectar los estudios y perspectivas empíricas del investigador, guiando la construcción de los resultados en tres secciones, que fueron descritas a partir de este momento. 


\section{ACERCA DE LA EVALUACIÓN DE LA ENSEÑANZA Y EL APRENDIZAJE}

Según el tiempo y el modelo de la sociedad, la evaluación tiene su trayectoria desde los primeros tiempos. Algunos autores como Soeiro y Aveline (1982) han expuesto como origen de los métodos de evaluación las actividades que el primer conglomerado humano realizó para, a través de rituales, hacer que los jóvenes indígenas estén listos para ser un adulto indígena. Para estos autores, estas prácticas se remontan a 360 .Bc considerado como un sistema de criterios utilizados por los chinos y griegos para permitir a los individuos para un trabajo determinado, permitiendo a todos los ciudadanos alcanzar posiciones de prestigio y poder.

Desde el principio ya había formas de evaluar, un acto utilizado por los chinos y griegos en el mercado laboral, esto sucedió inconscientemente, impuesto por el modelo cultural de la sociedad, con el objetivo de preparar y seleccionar lo mejor para mantener las posiciones de prestigio y poder. Este modelo de evaluación también se utilizó en China en el año 2200 a. C. (SOEIRO; AVELINE, 1982).

En Grecia, Sócrates sugirió una autoevaluación, "conocerse a sí mismo" como una manera de llegar a la verdad. Por lo tanto, este pensamiento filosófico se ha convertido en una forma de examen, es decir, autoevaluación, una acción de extrema importancia, actualmente, para la eficacia del proceso de enseñanza-aprendizaje.

Dar mayor énfasis a la trayectoria de la evaluación Despresbiteris (1999, p. 113) informa que en "[...] 2005 a. C. El emperador chino Shum examinó a sus oficiales cada tres años con el objetivo de promover o disparar, principalmente para asegurar a los hombres entrenados en defensa del estado". Otro ejemplo importante de cómo se desarrolló la evaluación es en las obras de Marx que [...]"el examen no es otro que el bautismo burocrático del conocimiento sagrado" (apud GARCIA, 2001, p. 29).

Así, el examen se enfatiza como burocracia, es decir, la parte escrita, en la que sólo se informa de lo apropiado para los aplicadores, en este caso está dirigido a la 
documentación y no al desarrollo intelectual utilizando lo que es mundano para los cambios y transformaciones al conocimiento sagrado.

También en el siglo XIII, Kraemer (2005) expone el uso del examen como una forma de evaluación a través de la disciplina organizada de los exámenes y el sistema de asignación de calificaciones, dando lugar a una ciencia llamada Docimologia. También explica que en el sentido etimológico la evaluación proviene del latín a + valere teniendo como significado atribución de valor y mérito al objetivo del estudio. El concepto utilizado en varios países ya mencionado tiene como objetivo seleccionar a las personas para realizar diversas funciones en el mercado laboral.

Con las transformaciones de la era tecnológica y la industrialización, a partir del siglo XX, la investigación y la evaluación sufrieron grandes influencias de las ciencias, se crearon numerosos procedimientos y teorías utilizadas hoy en día, como la Encuesta $y^{[4]}$ otros instrumentos: objetivos estandarizados y pruebas escritas.

Vianna (2005) señala que los primeros treinta años de vida social estadounidense en el siglo XX fueron influenciados por tres elementos desarrollados en principio para la gestión industrial: sistematización, estandarización y eficiencia, que finalmente afectó a toda la sociedad, incluso en el área educativa. Por lo tanto, había una gran preocupación con el mercado laboral, la eficiencia era el gran objetivo y, en consecuencia, desarrolla técnicas para identificar dónde los estudiantes no eran capaces de captar los conocimientos que se enseñan en el aula.

En la década de 1970, la evaluación terminó creando un paradigma positivista, que debería romperse con la construcción de nuevas formas de evaluación, aplicando las novedades que estaban de moda en el continente norteamericano. Así, se desarrollaron procedimientos para comparar el desempeño de los estudiantes con las pruebas y la composición de nuevos planes educativos incluyendo la evaluación como un proceso para medir este desempeño en las disciplinas. Para El Prado de Sousa (1998), fue a partir de la década de 1980 cuando la evaluación educativa se hizo más relevante en el contexto escolar. Fue entonces cuando la sociología comenzó a 
contribuir con investigaciones y estudios relevantes sobre el funcionamiento interno de la escuela.

El concepto de aprendizaje como objeto de evaluación es otro pilar en la defensa de una concepción de la evaluación educativa. El aprendizaje humano no se limita a la adquisición de conocimientos, habilidades, normas de comportamiento, "aséptico" de valores y sentido personal. El uso del concepto de aprendizaje para describir procesos cognitivos y adquisiciones, excluyendo "afectivo" y "contivo" es inconsistente porque distorsiona la realidad que se pretende afectar y evaluar la dimensión desconocida del conocimiento. Toda acción cognitiva tiene valor, conocimiento, habilidad, forma de comportamiento lleva un sentido personal, es un sujeto que aprende. La evaluación requiere tener el modelo de objetos desde una perspectiva integral, holística y multifacética que resalte la complejidad y la riqueza del aprendizaje. Argumentan que la necesidad de evaluar la enseñanza o la formación (GIBBS, 2003).

\section{LA IMPORTANCIA DE LA AUTOEVALUACIÓN PARA EL EDUCADOR IMPARCIAL}

La autoevaluación se ha considerado una estrategia muy eficaz para adoptar conceptos democráticos en el proceso de gestión escolar, haciendo que los ciudadanos, gerentes, maestros y toda la comunidad escolar participen en el proceso de asignación de recursos en la educación escolar (CAMILLONI, 1997).

El esquema de autoevaluación consiste en hacer que los procesos internos y externos en los que la escuela está participando puedan tener sus resultados medidos racionalmente y demostrar la eficiencia de la forma en que la erofeógico y la didáctica para analizar su planificación pedagógica y didáctica en las estrategias de enseñanza en el aula, por lo tanto, hay autoevaluación cuando se pueden identificar fortalezas (potencias) y frágiles (efemérides) en los resultados.

Los problemas de los temas de evaluación son abordar el debate sobre quién debe, puede y es capaz de participar en la evaluación. Lucha y defiende la necesidad y el derecho del estudiante universitario a participar en su evaluación, el valor educativo y 
de formación que se aporta a sí mismo y las demás asignaturas del proceso de enseñanza-aprendizaje. El yo y el hetero son facetas necesarias del trabajo individual y grupal en la educación superior, que refuerzan y expresan la relación entre el trabajo del grupo y la responsabilidad individual de cada tributo especial al mismo; con el fin de contribuir al desarrollo de la responsabilidad social de posgrado como requisito para la educación superior. Se establece como un propósito legítimo que invierte el evaluador evaluador.

La autoevaluación se produce a través de procesos que, cuando el profesor los realiza y los adopta, carecen de conocimiento sobre la información y los criterios que deben evaluarse porque fueron realizados por quienes los están evaluando, midiendo sus propios resultados y considerando si tales resultados propios fueron satisfactorios para el éxito del proceso de aprendizaje docente. En este caso, los maestros aplican pruebas de autoevaluación cada vez que se enseñan cursos, conferencias, capacitación y consejería en el aula y en otros espacios escolares.

La evaluación educativa, desde su origen, ya aporta a su protuberancia una complejidad, debido a los numerosos factores que deben tenerse en cuenta a la hora de evaluar a alguien o algo, y es importante destacar que hoy en día existe una conciencia de la sociedad brasileña sobre la relevancia del proceso de evaluación educativa.

Por lo tanto, la evaluación es necesaria, y continuamente, más aún cuando la evaluación se considera como un compromiso formal y una práctica informal. Desde la actualidad la evaluación se entiende como una acción indispensable en cualquier momento de la vida del ser humano, oponiéndose naturalmente al servicio de la educación, abordando las experiencias de aprendizaje, el desarrollo humano, la mejora de la calidad de vida, el bien-voluntad, la elevación de la autoestima y la valorización de las iniciativas entre las personas.

Garantizar la educación para todos está relacionado con el cambio de un currículo alejado de los problemas del mundo y los intereses de los propios estudiantes a un plan de estudios capaz de captar el interés tanto de los estudiantes como de los 
profesores, favoreciendo la comprensión de los fenómenos naturales, sociales y culturales, como un instrumento que predispone al cuestionamiento, el aprendizaje en la acción, la actitud de enfrentar la incertidumbre de complejidad, teniendo en cuenta los nuevos conocimientos, habilidades y valores.

El educador necesita reevaluar su praxis y didáctica buscando desarrollar un contenido más significativo y una metodología más participativa, de tal manera que reduzca la necesidad de utilizar la nota como instrumento de coerción. Esta tarea no es fácil en el contexto actual, esta actuación del educador tiene que ver con hacer frente a la alienación: es una lucha de perspectivas, de significados para el conocimiento y para la vida.

En este contexto, el LDB como guía jurídico de las políticas educativas tiene como objetivo recuperar a través de la escuela pública su papel en la formación de la ciudadanía, estando abierto a todos los estudiantes, sin discriminación, integrando la diversidad sociocultural y las diferencias individuales contribuyendo así a una socialización integradora. La educación para la ciudadanía apunta a una forma de afrontar nuevos problemas, permitiendo al mismo tiempo la integración de valores y temas transversales, en lugar de presentarlos como acciones específicas o aisladas, sin limitarse a aprender ciertos valores, comportamientos o actitudes, ya que el ciudadano necesita todo el conjunto de conocimientos y habilidades que le permitan participar activamente en la vida pública, sin el cual se le puede excluir o negar la ciudadanía.

Sin embargo, todavía hay muchos desafíos a los que se debe hacer frente para que la educación pública sea una condición para la cohesión social y la integración: más eficiencia en la gestión y más densidad en los contenidos; diálogo entre el conocimiento científico, los sistemas de interpretación del mundo y la sabiduría ancestral de los pueblos latinoamericanos; escuelas abiertas a la sociedad y sus necesidades; definición de habilidades básicas y fundamentales para la formación de los estudiantes, con la creación de sistemas de información y evaluación; mayor reconocimiento social al magisterio, dignificando y profesionalizando a los educadores. 


\section{UTILIZACIONES DE LA EVALUACION PARTICIPATIVA POR OBJETIVOS EN LA EDUCACION BASICA BRASILEÑA}

En el contexto actual, la evaluación que tenemos a veces se confunde con conceptos y calificaciones, y se realiza para clasificar a los estudiantes en aprobados y desaprobados. Hay minorías que pueden visualizar y realizar la evaluación con el fin de contribuir a la calificación del proceso educativo. La mayoría prefiere ordenar las calificaciones, porque piensan que es la forma más fácil, que por lo que tendrán menos trabajo.

Muchas escuelas evalúan a sus estudiantes a través de exámenes, prácticas que son de naturaleza positivista y técnica que no contemplan el aprendizaje en su conjunto, actuando de manera clasificatoria y excluyendo, porque aunque las calificaciones/conceptos son un registro necesario, debido al sistema educativo actual, no pueden representar la evaluación en sí, distorsionando el proceso de enseñanzaaprendizaje. En este tipo de evaluación que clasifica, la más saludable es el producto. Reflexionando, una educación basada en la memorización de contenidos.

Contrariamente a este tipo de evaluación que enfatiza la atribución de grados y clasificación, se puede evidenciar la evaluación cualitativa que se basa en un paradigma crítico y tiene como objetivo mejorar la calidad del sistema educativo. Considerando que su énfasis está en el proceso de superar las dificultades de los estudiantes. $Y$ esta es la evaluación que se desea actualmente, frente a tantos desafíos que la escuela pasa diariamente.

Actualmente existe un consenso sobre los nuevos métodos de evaluación que se están implementando en el proceso de enseñanza de la educación básica. Esta novedad en el ámbito de la Pedagogía ha generado la participación de los alumnos y ha aportado información y opiniones que pueden fomentar la transformación del conocimiento y la enseñanza, y culminar con la mejora de la calidad de la enseñanza con la inserción, por ejemplo, de la evaluación participativa por objetivos, estrategias de evaluación que coinciden con opiniones y visión personal -crítica o de superaciónde las estrategias que los profesores han adoptado en el aula. 
Esta técnica se ha utilizado para identificar resultados tangibles de los objetivos y la planificación pedagógica considerados por los profesores, identificando también puntos relacionados con las imágenes de rendimiento (mejora o empeoramiento) de los alumnos, así como identificando sus opiniones si se adoptan cambios en las prácticas pedagógicas actuales. Según Kraemer (2005), esta práctica tiene dos funciones importantes, con sus breves descripciones en el Gráfico 1.

Tabla Uno. Clasificación de las funciones de evaluación por objetivos.

\begin{tabular}{|c|c|}
\hline Clasificación & Funciones y características \\
\hline $\begin{array}{l}\text { Función de } \\
\text { diagnóstico }\end{array}$ & $\begin{array}{l}\text { Entre sus funciones, destacamos las opiniones de quienes están } \\
\text { siendo evaluados en relación con la gestión de las estrategias de } \\
\text { enseñanza del profesorado, por ejemplo; o incluso las } \\
\text { perspectivas de los estudiantes sobre las causas del bajo } \\
\text { rendimiento en el aula en una disciplina dada. Esta forma de } \\
\text { recopilar datos propone el uso de prepruebas, encuestas y } \\
\text { estrategias innovadoras, atrevidas y creativas (tormenta } \\
\text { cerebral), cuyo objetivo es ayudar en el diagnóstico de la } \\
\text { enseñanza y la adopción de la cultura de los cambios en las } \\
\text { estrategias pedagógicas presentes. }\end{array}$ \\
\hline $\begin{array}{lr}\text { Función de } & \text { duma, } \\
\text { clasificatorio } & \end{array}$ & $\begin{array}{l}\text { Entre sus principales características, destaca la adopción de } \\
\text { evaluaciones que puedan clasificar y tipificando la comprensión } \\
\text { sobre las pruebas elaboradas por los profesores, o de lo } \\
\text { contrario, la clasificación del estudiante al final del año escolar } \\
\text { (aprobada o desaprobada). Fue el más aceptado en las escuelas } \\
\text { para asegurar sintéticamente la construcción de percepciones } \\
\text { sobre la realidad escolar en el aula y al final de bimasters y } \\
\text { semestres. }\end{array}$ \\
\hline
\end{tabular}

Fonte: Kraemer (2005).

Con este enfoque, se puede inferir que la evaluación del rendimiento escolar debe practicarse como la atribución de una cualidad a los resultados de aprendizaje de los 
estudiantes, basada en sus aspectos esenciales y que como objetivo final, favorece una toma de decisiones que dirija el aprendizaje y, en consecuencia, el desarrollo del alumno.

Así, la evaluación diagnóstica se configura como un momento de comprensión de la etapa en la que el alumno tiene la distancia en la que se refiere a los objetivos articulados que deben alcanzarse por delante. La evaluación diagnóstica también favorece la participación social de todos los estudiantes como ciudadanos, ya que ya no tiene el carácter conservador y antidemocrático de la evaluación de clasificación que excluyó a muchas personas de la escuela.

En esta premisa, la evaluación diagnóstica tiene como objetivo identificar, verificar, investigar las dificultades y objetivos alcanzados para cambiar el tamaño de la acción educativa, y es un acto de procedimiento, llevando al educador a reflexionar sobre los resultados de su investigación, construyendo nuevas propuestas, metodologías, con miras a la calidad y el compromiso de la enseñanza, , para detectar los diversos momentos de su desarrollo, no limitarse al juicio sobre el éxito o fracaso de los estudiantes, entendiéndolo como un conjunto de actuaciones que tiene la función de guiar la intervención pedagógica, esto sucede de forma continua y sistemática a través de la interpretación cualitativa del conocimiento construido por el alumno.

El profesor utilizará la evaluación como un instrumento que le permite cuestionar constantemente sus propuestas en el proceso de enseñanza-aprendizaje, permitiendo al educador redirigir su práctica.

\section{CONCLUSIÓN}

Se considera que se respondió el problema del artículo, se alcanzó el objetivo general y se confirmó la hipótesis de que cualquier instrumento utilizado por los profesores y la escuela para realizar la evaluación, configura ciertos, establecer niveles de aprendizaje, reconocer lo mejor y lo peor, y sobre todo, adaptar la enseñanza a los estándares de excelencia instalados por la escuela. Las medidas estandarizadas han 
desviado el sentido democrático, crítico, constructivo y creativo de la evaluación, impidiendo el camino del aprendizaje efectivo.

También se han alcanzado los objetivos. En cuanto al objetivo de analizar la evaluación de la enseñanza y el aprendizaje, los autores analizados exponieron el origen de los métodos de evaluación y sus usos por parte de los chinos y griegos en el mercado laboral. La marca del siglo XIII fue la organización de exámenes de evaluación con la atribución de calificaciones, dando lugar a una ciencia llamada Docimologia. Hoy en día, se desarrollaron procedimientos para comparar el desempeño de los estudiantes con las pruebas y la composición de nuevos planes educativos incluyendo la evaluación como un proceso de medición de este desempeño en las disciplinas.

En relación con el segundo objetivo específico, que proponía analizar la importancia de la autoevaluación para el educador imparcial, se encontró en los estudios seleccionados que el esquema de autoevaluación consiste en hacer que los procesos internos y externos en los que la escuela está participando puedan medir sus resultados racionalmente y demostrar la eficiencia de la forma en que su planificación pedagógica y didáctica ha generado resultados satisfactorios en las estrategias de enseñanza en el aula por lo tanto, hay autoevaluación cuando se pueden identificar fortalezas (potencias) y frágiles (efemérides) en los resultados.

Por último, se logró el tercer objetivo específico, demostrando que los usos de la evaluación participativa por objetivos en la educación básica brasileña resultan en una manera contraria a la de la evaluación que da calificaciones y clases. Su énfasis está en el proceso de superar las dificultades de los estudiantes. Y esta es la evaluación que se desea actualmente, frente a tantos desafíos que la escuela pasa diariamente. Esta técnica se ha utilizado para identificar resultados tangibles de los objetivos y la planificación pedagógica considerados por los profesores, identificando también puntos relacionados con las imágenes de rendimiento (mejora o empeoramiento) de los alumnos. 


\section{REFERENCIAS}

CAMILLONI, A. R. W. Los obstáculos epistemológicos em la enseñanza. Barcelona: Gedisa, 1997.

DEPRESBITERIS, Léa. O desafio da avaliação da aprendizagem: dos fundamentos a uma proposta inovadora. $1^{\underline{a}}$ ed. São Paulo: Editora Pedagógica e Universitária, 1999.

GARCIA, Ronaldo Coutinho. Subsídios para organizar avaliações da ação governamental. Planejamento e Políticas Públicas, Brasília, n. 23, p. 7-70, jan. /jun. 2001.

GIBBS, G. Uso estratégico de la evaluación en el aprendizaje. In: Brown, S.; Glasner, A. Evaluar em la Universidad: problemas y nuevos enfoques. Madrid: Narcea, 2003, pp. 61-70.

KRAEMER, Maria Elisabeth Pereira. A avaliação da aprendizagem como processo construtivo de um novo fazer. Avaliação: Revista da Avaliação da Educação Superior, v. 10, n. 2, 137-147, 2005.

SOEIRO, L \& AVELINE, S. Avaliação Educacional. Porto Alegre: Editora. Sulina, 1982. VIANNA, Heraldo Marelim. Fundamentos de um Programa de Avaliação Educacional. Brasília: Liber Livro Editora, 2005.

4. Testes normativos usados para avaliar a eficiência da Instituição escolar.

Publicado: Septiembre, 2019.

Aprobado: Noviembre, 2019. 